\title{
Leucemia linfoblástica aguda en pacientes portadores de síndrome de Down
}

\author{
BENIGNO MONTENEGRO R. ${ }^{1}$, MYRIAM CAMPBELL B. ${ }^{2,3,4}$, NATALIE RODRÍGUEZ Z. ${ }^{2,3}$ \\ 1. Becado. Programa de Especialización en Hemato-Oncología Pediátrica. Escuela de Postgrado. Facultad de Medicina. \\ Universidad de Chile. \\ 2 Unidad de Hemato-Oncología. Hospital de Niños Roberto del Río. \\ 3 Departamento de Pediatría y Cirugía Infantil. Campus Norte. Facultad de Medicina. Universidad de Chile. \\ 4 Coordinador Protocolo Leucemia Linfoblástica Aguda (PINDA), Ministerio de Salud.
}

\begin{abstract}
Acute Lymphoblastic Leukemia in Children with Down`s Syndrome

Background: Children with Down's Syndrome (DS) present a higher incidence of Acute Lymphoblastic Leukemia (ALL) with more complications and shorter survival than healthy children. Objective: To describe clinical characteristics, laboratory and treatment results in patients with DS and ALL. Patients and Method: Retrospective analysis of 42 DS and ALL patients treated in three consecutive trials $(1992,1996,2002)$ from the Pediatric National Cancer Program (PINDA). Clinical data, immunophenotype, cytogenetics and treatment results were analyzed. Results: There was no difference by age or gender, no patient presented LLA-T, $t$ $(9 ; 22)$ o $t(4 ; 11)$. Of the 42 patients, 38 patients went into remission, 10 relapsed (26,3\%), 11 died because of infection, none died from other toxicity. Survival at 5 years was $35 \pm 9 \%$ (median of follow-up was $50 \mathrm{mo}$ ), similar for all protocols $(\mathrm{p}=0,61)$. Conclusion: The group of patients with ALL and DS evaluated was not associated with classic treatment resistance factors. The relapse rate was not increased, if compared with non DS ALL patients; in this group the infections were the determinant factor for a lower survival. These patients can be treated with the current trials but they require a detailed infection care.
\end{abstract}

(Key words: Down Syndrome, acute lymphoblastic leukaemia, chemotherapy).

Rev Chil Pediatr 2012; 83 (1): 58-67

\section{RESUMEN}

Los niños con Síndrome de Down (SD) tienen mayor incidencia de leucemia linfoblástica aguda (LLA) con más complicaciones y menor sobrevida que los niños sin SD. Objetivo: Describir características clínicas, de laboratorio y resultados de tratamiento en niños con SD y LLA. Pacientes y Método: Análisis retrospectivo de 42 pacientes con LLA y SD tratados en 3 protocolos consecutivos (1992, 1996 y 2002) del Programa Nacional de Cáncer Infantil (PINDA). Se analizaron datos clínicos, de laboratorio, inmunofenotipo, citogenética y resultados de tratamiento. Resultados: La distribución por género o grupo etario no mostró diferencias, ningún paciente presentó LLA-T, t (9;22) o t (4;11). De los 38 pacientes que remitieron, 10 recayeron (26,3\%),

Trabajo recibido el 01 de abril de 2011, devuelto para corregir el 09 de mayo de 2011, segunda versión el 21 de noviembre de 2011, aceptado para publicación el 18 de diciembre de 2011.

Correspondencia a:

Dr. Benigno Montenegro R.

E-mail: benigno.montenegro@redsalud.gov.cl 
fallecieron por infección 11/42 (26,2\%). Ninguno falleció por otra toxicidad. La sobrevida libre de eventos global a 5 años fue $35 \pm 9 \%$ (mediana de seguimiento 50 meses), siendo similar en los diferentes protocolos usados ( $\mathrm{p}=0,61)$. Conclusión: Los pacientes evaluados con SD y LLA no presentaron factores clásicos de resistencia a tratamiento. No se observó mayor frecuencia de recaída respecto a los pacientes con LLA sin SD y la menor sobrevida en este grupo fue determinada por infecciones. Estos pacientes pueden ser tratados con los protocolos actuales pero requieren un manejo precoz e intensivo de las infecciones.

(Palabras clave: Síndrome de Down, leucemia linfoblástica aguda, quimioterapia).

Rev Chil Pediatr 2012; 83 (1): 58-67

\section{Introducción}

Los pacientes con síndrome de Down (SD) son un grupo especial de niños que además de presentar alteraciones cardíacas, digestivas y/o endocrinas, tienen mayor riesgo que la población general de desarrollar leucemia, siendo 10 a 20 veces más frecuente en ellos en comparación con niños sin SD. Además en la población pediátrica es mucho más frecuente la Leucemia Linfoblástica Aguda (LLA) que la Leucemia Mieloide Aguda (LMA), en cambio los pacientes con SD tienen frecuencias similares de ambas ${ }^{1-3}$.

Existen ciertas condiciones propias del paciente con SD que lo predisponen a presentar leucemia y que han demostrado tener implicancia pronóstica. La asociación entre trisomía 21 y la mutación del factor de transcripción hematopoyético GATA 1 (localizado en el cromosoma X) promueve la proliferación de los megacariocitos inmaduros generando una expansión clonal de estas células. Estos pacientes tienen una mayor posibilidad de desarrollar trastorno mieloproliferativo transitorio (TMT) y eventualmente LMA (generalmente megacariocítica). Los pacientes con SD y LMA que presentan la mutación de GATA 1 tienen mejor respuesta al tratamiento y mejor pronóstico que la población general. Sin embargo, en los pacientes con SD y LLA no se ha descrito esta mutación, observándose peor pronóstico que en los pacientes sin SD en este tipo de leuce$\mathrm{mia}^{4-6}$.

El tratamiento de la LLA en niños ha tenido importantes avances, debido entre otros, a la mejoría de los esquemas de tratamiento con diferentes combinaciones de antineoplásicos (protocolos) obteniendo una sobrevida libre de eventos (SLE) a 5 años entre $78 \% \pm$ 0,9 y $83 \% \pm 2$, según reportes de los grupos internacionales más reconocidos ${ }^{7-10}$. En Chile, los resultados del grupo colaborativo nacional PINDA (Programa Infantil Nacional de Drogas Antineoplásicas) del Ministerio de Salud son similares, con una SLE a 5 años de $60 \pm$ $2 \%, 67 \pm 2 \%$ y $73 \pm 2 \%$ en los protocolos 87 , 92 y 96, respectivamente, y en protocolo 2002 SLE a 4 años de $79 \pm 2 \%{ }^{11,12}$.

La SLE en pacientes con SD y LLA es menor, variando entre 23 a $71 \%$ según diferentes estudios. Este hecho no se ha relacionado con una mayor incidencia de factores de riesgo descritos en LLA, sino más bien con la toxicidad asociada a terapia y la predisposición a infecciones más graves ${ }^{13,14}$.

El objetivo del estudio es describir las características clínicas, del inmunofenotipo y citogenética de los pacientes con SD y LLA tratados con protocolos PINDA y evaluar los resultados y complicaciones del tratamiento.

\section{Pacientes y Método}

\section{Población}

De un total de 1754 pacientes menores de 15 años con diagnóstico de LLA registrados y tratados entre los años 1992 y 2009, en 3 protocolos PINDA consecutivos (92, 96 y 2002), se evaluaron 42 niños con LLA y SD. El Protocolo 92 incluyó pacientes entre 1992 y 1995; el protocolo 96 se desarrolló entre 1996 y 2001 y el protocolo 2002, desde el año 2002 hasta la fecha. Los protocolos fueron aprobados por los comités de ética respectivos y se obtuvo consentimiento informado de todos los padres para la aplicación del tratamiento antineoplá- 
sico y la evaluación de los resultados obtenidos. El diagnóstico de SD fue establecido por características fenotípicas y por cariograma constitucional. El diagnóstico de LLA se basó en los estudios morfológicos y de inmunofenotipo en médula ósea de acuerdo a estándares internacionales ${ }^{15}$. El tratamiento en cada caso fue establecido de acuerdo a categorización de riesgo considerando: edad, leucocitos al diagnóstico, presencia de alteraciones citogenéticas y respuesta al tratamiento, según se describe en las tablas 1 y 2 .

\section{Tratamiento}

Los protocolos PINDA 1992, 1996 y 2002 están basados en los del grupo de estudio internacional Berlin-Frankfurt-Munster (BFM) con mínimas adaptaciones. En la tabla 3 se muestran las distintas fases de tratamiento utilizadas. A diferencia de los protocolos BFM que utilizan metotrexato (MTX) $5 \mathrm{gr} / \mathrm{m}^{2}$, en el protocolo PINDA 1992 los pacientes de riesgo estándar recibían $1 \mathrm{gr} / \mathrm{m}^{2}$ de MTX y los pacientes de riesgo intermedio se randomizaban para recibir 1 ó $2 \mathrm{gr} / \mathrm{m}^{2}$. En los pacientes de alto riesgo en el protocolo 1992 no se administraba la fase 2 del protocolo I y recibían 9 ciclos de intensificación con múltiples drogas (entre ellas MTX 2 gr $/ \mathrm{m}^{2}$ ). En los protocolos 1996 y 2002 todos los pacientes recibieron MTX $2 \mathrm{gr} / \mathrm{m}^{2}$. En riesgo alto se redujeron a 6 los ciclos de intensificación en el protocolo 1996 y a 3 en el protocolo 2002, incorporando la fase 2 del protocolo I. En el protocolo iniciado el 2002 se evaluó en forma randomizada distintos tipos de reinducción para todos los grupos de riesgo. La randomización fue por blocks permutados, por método computacional y centralizada. Desde el año 1999 el trasplante de precursores hematopoyéticos (TPH) se indicó en pacientes con LLA de alto riesgo que presenten algunos factores específicos de mal pronóstico como: mala respuesta a prednisona asociada con leucocitosis mayor a 100 000/dL, presencia de translocación (9;22) o falta de remisión al día 33 de tratamiento, entre otras. La condición de SD per se no es indicación ni contraindicación de TPH.

\section{Análisis estadístico}

Las diversas características clínicas y de laboratorio se expresaron como frecuencia relativa respecto del grupo total de pacientes con LLA y SD.

La SLE fue calculada desde el momento del diagnóstico hasta el primer evento de falla en el tratamiento (muerte o recaída) o la última

Tabla 1. Definición de grupos de riesgo

\begin{tabular}{|ll|}
\hline Grupo de Riesgo & Definición \\
\hline Estándar & BRP y Edad $1-5$ años y Leucocitos al diagnóstico $<20000$ y M1-M2 día 15 y RC al día 33 \\
\hline Intermedio & $\begin{array}{l}\text { BRP y Edad } \geq 6 \text { años y/o Leucocitos al diagnóstico } \geq 20000 \text { y M1-M2 día } 15 \text { y RC día } 33 . \\
\text { Pacientes R. Estándar con M3 al día } 15 \text { y RC día } 33\end{array}$ \\
\hline Alto & MRP o Riesgo intermedio + M3 día 15 o no RC día 33 o t $(4 ; 11)$ o t (9;22) \\
\hline
\end{tabular}

BPR: buena respuesta a prednisona, MRP: Mala respuesta a prednisona, M1: médula ósea con < 5\% blastos, M2: médula ósea con 5-25\% blastos, M3: médula ósea con > 25\% blastos, RC: remisión completa.

Tabla 2 . Definición de respuesta a tratamiento

Respuesta a prednisona: Definida como el número de linfoblastos en sangre periférica después de 7 días de prednisona. Se considera como buena respuesta a prednisona (BRP): blastos $<1000 / \mu \mathrm{L}$ y mala respuesta prednisona (MRP): blastos $>1000 / \mu \mathrm{L}$

El recuento de blastos en la médula ósea al día 15:

Médula ósea M1 < de $5 \%$ de blastos

Médula ósea M2 entre $5-25 \%$ de blastos

Médula ósea $\mathrm{M} 3>25 \%$ blastos

Remisión completa al día 33 (RC d33): Menos de 5\% de blastos en médula ósea al día 33 de iniciada la inducción y sin evidencia de enfermedad clínica 
Tabla 3. Drogas utilizadas en las diferentes fases de tratamiento LLA

\begin{tabular}{|c|c|c|}
\hline Droga & Dosis & Día de administración \\
\hline \multicolumn{3}{|l|}{ Inducción protocolo I } \\
\hline \multicolumn{3}{|l|}{ FASE 1} \\
\hline Prednisona (o) & $60 \mathrm{mg} / \mathrm{m}^{2}$ & $1-28$ \\
\hline Vincristina (iv) & $1,5 \mathrm{mg} / \mathrm{m}^{2}$ & $8,15,22,29$ \\
\hline Daunorubicina (iv) * & $40 \mathrm{mg} / \mathrm{m}^{2}$ & $8,15,22,29$ \\
\hline L-Asparraginasa (iv) \& & $10000 \mathrm{IU} / \mathrm{m}^{2}$ & $19,22,25,28,31,34,37,40$ \\
\hline \multicolumn{3}{|l|}{ FASE 2} \\
\hline Ciclofosfamida (iv) & $1000 \mathrm{mg} / \mathrm{m}^{2}$ & $43-71$ \\
\hline Citarabina (iv) & $75 \mathrm{mg} / \mathrm{m}^{2}$ & $45-48,52-55,59-62,66-69$ \\
\hline 6 Mercaptopurina (o) & $60 \mathrm{mg} / \mathrm{m}^{2}$ & $43-70$ \\
\hline Metotrexato (IT ) & $12 \mathrm{mg}$ * & $1,45,59$ \\
\hline \multicolumn{3}{|c|}{ Consolidación protocolo M } \\
\hline 6 Mercaptopurina (o) & $25 \mathrm{mg} / \mathrm{m}^{2}$ & $1-56$ \\
\hline Metotrexato (24 h iv) & $1-2 \mathrm{~g} / \mathrm{m}^{2}$ & $8,22,36,50$ \\
\hline Metotrexato (IT) & $12 \mathrm{mg}$ * & $8,22,36,50$ \\
\hline \multicolumn{3}{|l|}{ Reinducción protocolo II } \\
\hline Dexametazona (o) & $10 \mathrm{mg} / \mathrm{m}^{2}$ & $1-21$ \\
\hline Vincristina (iv) & $1,5 \mathrm{mg} / \mathrm{m}^{2}$ & $8,15,22,29$ \\
\hline Doxorubicina (iv) & $30 \mathrm{mg} / \mathrm{m}^{2}$ & $8,15,22,29$ \\
\hline L-Asparraginasa (iv) & $10000 \mathrm{IU} / \mathrm{m}^{2}$ & $8,11,15,18$ \\
\hline Ciclofosfamida (iv) & $1 \mathrm{~g} / \mathrm{m}^{2}$ & 36 \\
\hline Citarabina(iv) & $75 \mathrm{mg} / \mathrm{m}^{2}$ & $38-41,45-48$ \\
\hline 6 Tioguanina (o) & $60 \mathrm{mg} / \mathrm{m}^{2}$ & $36-49$ \\
\hline Metotrexato (IT) & $12 \mathrm{mg} *$ & 38,45 \\
\hline \multicolumn{3}{|c|}{ Reinducción protocolo III } \\
\hline Dexametasona (o) & $10 \mathrm{mg} / \mathrm{m}^{2}$ & $1-14$ \\
\hline Vincristina (iv) & $1,5 \mathrm{mg} / \mathrm{m}^{2}$ & 1,8 \\
\hline Doxorubicina (iv) & $30 \mathrm{mg} / \mathrm{m}^{2}$ & 1,8 \\
\hline L-Asparraginasa (iv) & $10000 \mathrm{IU} / \mathrm{m}^{2}$ & $1,4,8,11$ \\
\hline Ciclofosfamida (iv) & $1 \mathrm{~g} / \mathrm{m}^{2}$ & 15 \\
\hline Citarabina(iv) & $75 \mathrm{mg} / \mathrm{m}^{2}$ & $17-20,24-27$ \\
\hline 6 Tioguanina (o) & $60 \mathrm{mg} / \mathrm{m}^{2}$ & $36-49$ \\
\hline Metotrexato (IT) & $12 \mathrm{mg}^{*}$ & 38,45 \\
\hline \multicolumn{3}{|l|}{ Mantención } \\
\hline Metotrexato (o) & $20 \mathrm{mg} / \mathrm{m}^{2}$ & Una vez por semana \\
\hline 6 Mercaptopurina (o) & $50 \mathrm{mg} / \mathrm{m}^{2}$ & Diario \\
\hline
\end{tabular}

*dosis MTX intratecal según edad (o):oral, (iv): intravenoso, (IT): intratecal. *en protocolos 96 y 2002 se disminuyó a $30 \mathrm{mg} / \mathrm{m} 2$ por dosis. ${ }^{\&}$ en protocolos 96 y 2002 se usó 8 dosis de 5000 uds e.v. entre día 11 y 33. fecha de seguimiento del paciente. El método de Kaplan-Meier fue utilizado para estimar la sobrevida en cada uno de los protocolos y los errores estándar correspondientes fueron calculados usando la fórmula de Greenwoods. Se utilizó el método de log rank test $\left(\chi^{2}\right)$ para comparar las curvas de sobrevida de cada protocolo.

\section{Resultados}

\section{Hallazgos clínicos y de laboratorio}

De un total de 1754 niños tratados en tres protocolos LLAPINDA consecutivos, 42 (2,4\%) fueron portadores de SD. La distribución en cada protocolo correspondió a 13/407 (LLA-PINDA 1992), 11/722 (LLA-PINDA 96) y 18/625 (LLA-PINDA 2002).

La distribución según género, edad, características clínicas, de laboratorio y categorización según riesgo se muestran en la tabla 4 . No hubo pacientes menores de 1 año. Al diagnóstico el 47,6\% presentaba hepatomegalia, 28,6\% esplenomegalia y 7,1\% masa mediastínica. El compromiso del SNC al diagnóstico fue de un 7,1\%, y no hubo compromiso testicular. Ningún paciente presentó comorbilidades que contraindicaran el tratamiento indicado.

De los 42 pacientes estudiados sólo se contó con estudio citogenético de los blastos en 29 , ya que éste se implementó a partir del año 1996. El 17,2\% (5/29) presentó hiperdiploidía, 10,3\% (3/29) presentó alteraciones citogenéticas múltiples, 3,5\% (1/29) presentó translocación $(8 ; 14)$ y en un $69 \%$ (20/29) no se obtuvieron mitosis para el análisis o presentaban trisomía 21 constitucional. En el grupo 
analizado no se encontró t $(9 ; 22)$ ni t $(4 ; 11)$. En relación a la categorización de riesgo, 5 pacientes se consideraron de riesgo alto: en 4 pacientes por mala respuesta a prednisona, la que se asoció con hiperleucocitosis en tres de ellos y un paciente presentó hiperleucocitosis asociada con más de $25 \%$ de blastos en médula ósea al día 15 de tratamiento.

\section{Respuesta a tratamiento}

En la tabla 5 se muestra el resultado de acuerdo a cada protocolo de tratamiento, con
20 niños que permanecen en remisión. Un paciente no remite al día 33 y fallece por leucemia sin obtener remisión luego de 5 ciclos intensivos. Tres pacientes fallecen por infección antes de remitir.

De los 38 pacientes que remitieron 10 recaen (26,3\%), la mayoría en médula ósea $(8 / 10)$. En ocho pacientes el momento de la recaída fue después de terminar el tratamiento (entre $5 \mathrm{~m}$ y 2 años 7 meses). De los 5 pacientes categorizados como de riesgo alto, 3 tenían indicación de TPH. De ellos, uno fallece por

Tabla 4. Características de pacientes con LLA con y sin SD

\begin{tabular}{|c|c|c|c|c|c|c|c|}
\hline & \multicolumn{2}{|c|}{ LLA con SD } & \multicolumn{2}{|c|}{ LLA $\sin$ SD } & \multicolumn{2}{|c|}{ Total } & \multirow[b]{2}{*}{$\mathbf{p}$} \\
\hline & $\mathbf{n}$ & $\%$ & $\mathbf{n}$ & $\%$ & n & $\%$ & \\
\hline & 42 & 2,4 & 1712 & 97,6 & 1754 & 100 & \\
\hline $\begin{array}{l}\text { Género } \\
\text { Femenino } \\
\text { Masculino }\end{array}$ & $\begin{array}{l}19 \\
23\end{array}$ & $\begin{array}{l}45,2 \\
54,8\end{array}$ & $\begin{array}{l}747 \\
965\end{array}$ & $\begin{array}{l}43,6 \\
56,4\end{array}$ & $\begin{array}{l}766 \\
988\end{array}$ & $\begin{array}{l}43,7 \\
56,3\end{array}$ & 0,25 \\
\hline $\begin{array}{l}\text { Edad } \\
\qquad \begin{array}{l}<1 \text { año* } \\
1-6 \text { años } \\
>6 \text { años }\end{array}\end{array}$ & $\begin{array}{r}0 \\
19 \\
23\end{array}$ & $\begin{array}{r}-- \\
45,2 \\
54,8\end{array}$ & $\begin{array}{r}17 \\
950 \\
745\end{array}$ & $\begin{array}{r}1,0 \\
55,5 \\
43,5\end{array}$ & $\begin{array}{r}17 \\
969 \\
768\end{array}$ & $\begin{array}{r}1,0 \\
55,2 \\
43,8\end{array}$ & 0,25 \\
\hline $\begin{array}{l}\text { Leucocitos diagnóstico } \\
\quad<20000 \\
\quad 20000-50000 \\
>50000\end{array}$ & $\begin{array}{r}27 \\
3 \\
12\end{array}$ & $\begin{array}{r}64,3 \\
7,1 \\
28,6\end{array}$ & $\begin{array}{r}1140 \\
258 \\
314\end{array}$ & $\begin{array}{l}66,6 \\
15,0 \\
18,4\end{array}$ & $\begin{array}{r}1167 \\
261 \\
326\end{array}$ & $\begin{array}{l}66,5 \\
14,9 \\
18,6\end{array}$ & 0,18 \\
\hline $\begin{array}{l}\text { Inmunofenotipo } \\
\text { LLA no T } \\
\text { LLA-T } \\
\text { No estudiado }\end{array}$ & $\begin{array}{r}40 \\
0 \\
2\end{array}$ & $\begin{array}{l}95,2 \\
0 \\
4,8\end{array}$ & $\begin{array}{r}1423 \\
168 \\
121\end{array}$ & $\begin{array}{r}83,1 \\
9,8 \\
7,1\end{array}$ & $\begin{array}{r}1463 \\
168 \\
123\end{array}$ & $\begin{array}{r}83,4 \\
9,6 \\
7,0\end{array}$ & $<0,05$ \\
\hline $\begin{array}{l}\text { Categorización según riesgo } \\
\text { Estándar } \\
\text { Intermedio } \\
\text { Alto }\end{array}$ & $\begin{array}{r}11 \\
26 \\
5\end{array}$ & $\begin{array}{l}26,2 \\
61,9 \\
11,9\end{array}$ & $\begin{array}{l}575 \\
935 \\
202\end{array}$ & $\begin{array}{l}33,6 \\
54,6 \\
11,8\end{array}$ & $\begin{array}{l}586 \\
961 \\
207\end{array}$ & $\begin{array}{l}33,4 \\
54,8 \\
11,8\end{array}$ & 0,20 \\
\hline
\end{tabular}

*Desde 1999 los menores de 1 año se tratan con protocolo especial para lactantes.

Tabla 5. Resultados de tratamiento en niños con LLA y SD

\begin{tabular}{|c|c|c|c|c|c|}
\hline & Prot 92 & Prot 96 & Prot 2002 & Total & $\%$ \\
\hline n pacientes & 13 & 11 & 18 & 42 & 100 \\
\hline Fallece en inducción por infección & 1 & 0 & 2 & 3 & 7 \\
\hline No remite y fallece por LLA & 1 & 0 & 0 & 1 & \\
\hline Remite & 11 & 11 & 16 & 38 & 90,5 \\
\hline Fallece en remisión por infección & 2 & 2 & 4 & 8 & 21,0 \\
\hline Recae & 5 & 3 & 2 & 10 & 26,3 \\
\hline Permanece en remisión & 4 & 6 & 10 & 20 & 47,6 \\
\hline
\end{tabular}


leucemia sin remitir, un segundo no tuvo donante y está en remisión y el tercero recibió un TPH alogénico y está en remisión. Los otros dos no tenían indicación de TPH y fallecen por infección después de remitir.

En protocolo 2002 se randomizaron 14 pacientes en la fase de reinducción, 6 niños recibieron protocolo III y de ellos uno recae. De los 9 que recibieron protocolo II, tres fallecieron por infección y uno recae. En cuánto a la evolución según el estudio citogenético, de los 5 niños con hiperdiplodía, uno recae, uno fallece por infección y 3 permanecen en remisión, al igual que el paciente con t $(8 ; 14)$. De los 3 niños con alteraciones genéticas múltiples uno fallece por infección y dos continúan en remisión; mientras que del grupo de 20 niños sin mitosis o sólo trisomía 21, fallecieron 6 por infección, 4 recaen y 10 permanecen en remisión.

\section{Toxicidad e infecciones}

En ésta serie hubo 11 pacientes fallecidos por infección (26,2\%), 3 de ellos en el primer mes de tratamiento, antes de remitir (2 por sepsis, 1 por neumonía) y 8 estando en remisión completa (6 por sepsis, 1 por neumonía, 1 por infección sin microorganismo aislado). La etapa de tratamiento en que fallecen después de obtener remisión es la siguiente: en protocolo
I un paciente, en protocolo II cuatro pacientes, uno en intensificación, uno en mantención y un paciente fallece por neumonía seis meses después de terminado el tratamiento.

En relación al uso de MTX, 6 pacientes no recibieron ninguna dosis por complicaciones previas (1 recaída y 5 fallecidos), ocho pacientes del protocolo 92 recibieron MTX $1 \mathrm{~g} / \mathrm{m}^{2}$ endovenoso en infusión continua (incluyendo 4 randomizados), 25 recibieron $2 \mathrm{gr} / \mathrm{m}^{2}$ (incluyendo 2 pacientes randomizados en protocolo 92), tres recibieron $5 \mathrm{gr} / \mathrm{m}^{2}$. No se observó toxicidad renal ni hepática. En 5 pacientes $(16,1 \%)$ se observó mucositis severa grado III (ulceración dolorosa, con imposibilidad de ingesta oral) o IV (requerimiento de alimentación parenteral) según el criterio común de toxicidad del $\mathrm{NCI}^{16}$. Uno de ellos fallece por sepsis posterior a intensificación con $5 \mathrm{gr} / \mathrm{m}^{2}$. Sólo un paciente presentó enteropatía neutropénica recuperándose satisfactoriamente.

\section{Resultado del tratamiento y sobrevida}

Con una mediana de observación de 50 meses, la SLE global a 5 años fue de $35 \pm 9 \%$ (figura 1). Los pacientes tratados en el protocolo 92 tuvieron una SLE de $30 \pm 12,8 \%$, en el protocolo 96 SLE $42 \% \pm 19$, 8 y en el protocolo 2002 SLE 41\% \pm 15,7 (figura 2). Al comparar la SLE de estos tres protocolos entre sí, me-

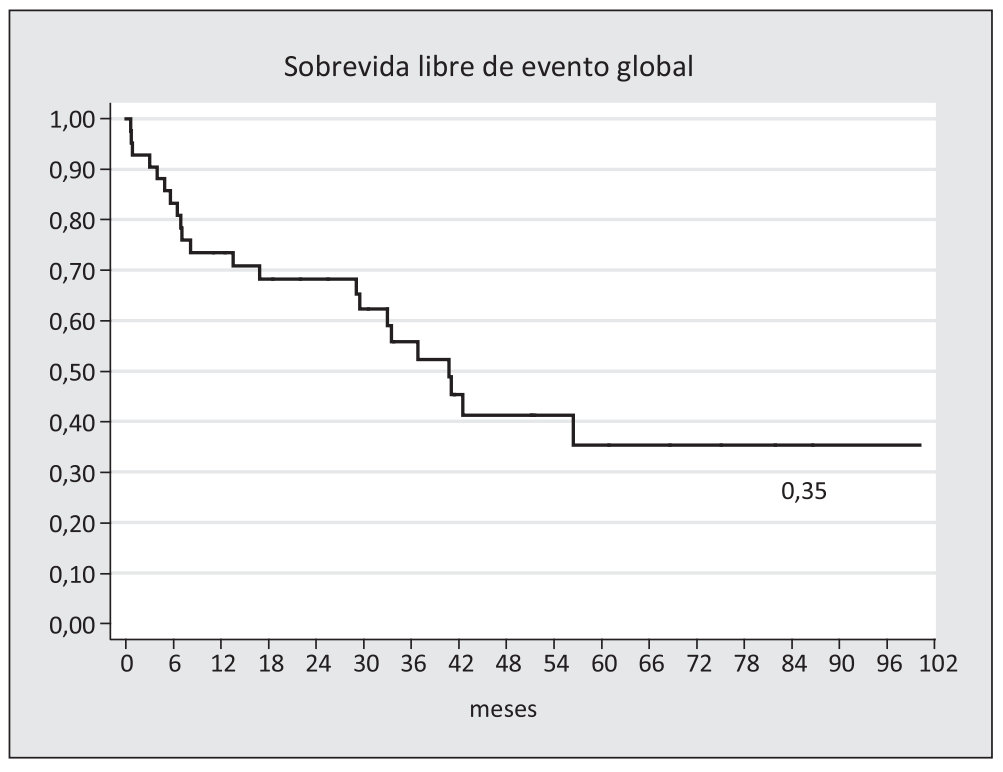

Figura 1. SLE a 5 años en el grupo total en niños con SD y LLA ( según Kaplan Meier). 


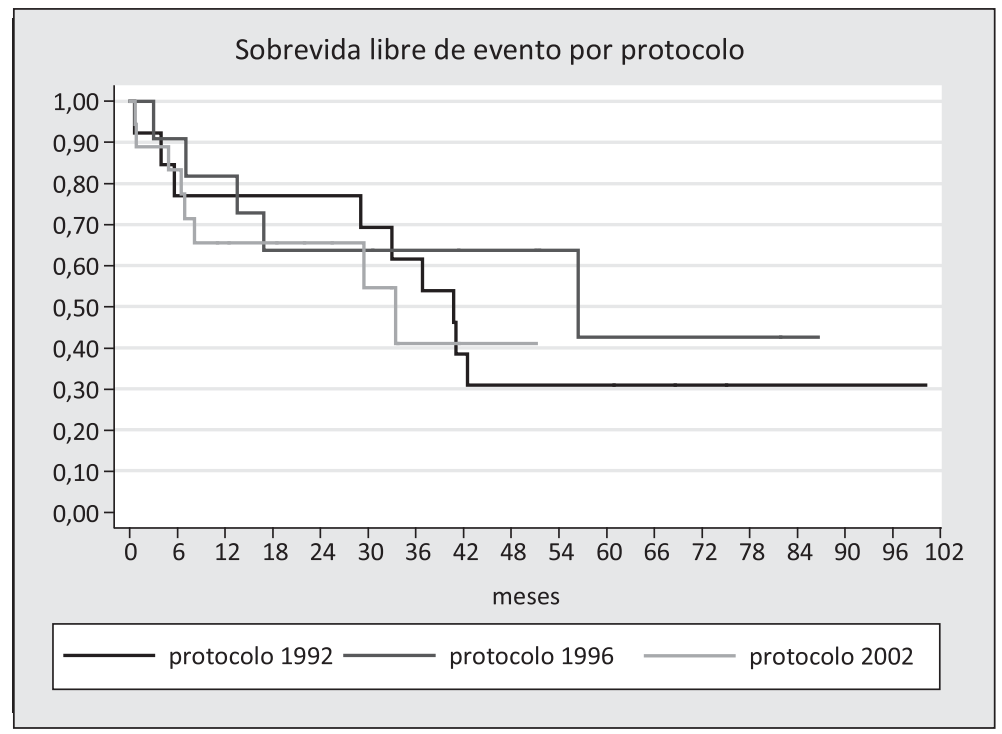

Figura 2. SLE a 5 años por protocolo en niños con SD y LLA (según Kaplan Meier). diante el método log rank test $\left(\chi^{2}\right)$, las diferencias no fueron significativas $(p=0,61)$. No se registraron pacientes con un segundo cáncer en el grupo.

\section{Discusión}

En nuestro estudio la incidencia de SD en pacientes con LLA fue de un 2,4\%, similar a la proporción reportada en la literatura, la que varía de $1,6 \%-2,1 \%{ }^{4-6}$.

Destaca la baja incidencia de compromiso mediastínico (2.4\%) y la ausencia de compromiso testicular, a diferencia de niños con LLA sin SD publicados por el grupo PINDA y $\mathrm{BFM}^{7,11}$.

En los pacientes con LLA sin SD la frecuencia de LLA T oscila entre 10-15,5\%. En este grupo de LLA con SD destaca la ausencia de LLA T, con una proporción más alta de LLA B común respecto a otros trabajos. Estos hallazgos explicarían la baja frecuencia de masa mediastínica que se observó en este grupo de pacientes $4,7,11$.

En los 29 casos en que se realizó estudio citogenético, no se encontraron translocaciones $t$ $(4 ; 11)$ y t $(9 ; 22)$, las que se asocian a alto riesgo de recaída o falla de tratamiento. Estos hallazgos ya han sido descritos en series previas, confirmando que los pacientes con SD rara vez presentan alteraciones genéticas asociadas a mal pronóstico. La asociación entre hiperdiploidía y pronóstico favorable en LLA es conocida y se explica fundamentalmente por un incremento en la sensibilidad frente a antimetabolitos como el MTX (por aumento del transportador de folatos reducidos) y por una mayor sensibilidad a los corticoides (mecanismo no totalmente aclarado). La frecuencia de 26\% (5/19) de hiperdiploidía en el grupo de niños con SD evaluado es similar a la población de pacientes con LLA sin SD, a diferencia de estudios que describen una frecuencia menor de hiperdiploidía a lo que atribuyen la menor probabilidad de curación. ${ }^{17-20}$. Esta disparidad puede ser explicada por el tamaño de la muestra estudiada.

Ya que los niños con SD y LLA no comparten las alteraciones genéticas reconocidas clásicamente como de mayor riesgo de recaída, actualmente se está estudiando la presencia de marcadores a nivel molecular más específicos en estos pacientes, como la presencia del gen JAK2 que transcribe una tirosina kinasa que activaría el sistema JAK/STAT favoreciendo la inmortalización de las células malignas en LLA y SD, lo que determina menor sensibilidad a quimioterapia en este tipo de leucemia. La importancia de este hallazgo es que podrían 
utilizarse en forma terapéutica inhibidores específicos de esta tirosina kinasa ${ }^{21}$.

En la categorización en grupos de riesgo, observamos que la mayoría de estos pacientes fueron incluidos en el grupo de riesgo intermedio, similar a lo reportado en trabajos del grupo BFM, AEIOP y a la población de niños con LLA sin $\mathrm{SD}^{22,23}$.

Al comparar la SLE de los niños con LLA y $\mathrm{SD}$ en los diferentes protocolos usados no hubo diferencia estadísticamente significativa, probablemente debido al número reducido de pacientes de esta muestra. La SLE para el grupo total de niños con LLA y SD es inferior a la reportada para niños con LLA sin SD ${ }^{7-12}$.

La frecuencia de recaídas de un 26,3\% es similar a la observada en pacientes con LLA sin SD, siendo la recaída en médula ósea la más frecuente en ambos grupos, como se ha descrito en estudios AEIOP y BFM ${ }^{7,10}$. En cambio la frecuencia de fallecimientos por infección en los niños con LLA sin SD, de 2 a $4 \%^{7,10}$, es menor que el $26 \%$ observado en este grupo de niños con LLA y SD. La menor SLE observada en este grupo de niños con SD y LLA evaluados esta dada por mayor mortalidad asociada a infecciones, como ha sido descrito ${ }^{22,23}$.

Las muertes por infección se presentaron en distintas fases del tratamiento y no se observaron con mayor frecuencia en aquellas en las que se utilizó MTX. Sólo un paciente de la serie fallece por sepsis posterior a mucositis secundaria a la administración de dosis altas de MTX (5 gr por $\mathrm{m}^{2}$ ). Ningún paciente tenía enfermedades recurrentes o malformaciones asociadas a SD que hubieran condicionado la mayor frecuencia de infecciones.

El MTX es una droga que se asocia a mayor toxicidad en pacientes con SD debido a que estos niños presentan un aumento de la cistationina-sintetasa, que inhibe la recuperación efectiva de los niveles de folatos para la síntesis de ácidos nucleicos. Además se ha descrito un incremento en los niveles del transportador de folato reducido (RFC) que es el responsable del transporte de MTX al interior de la célula, cuyo gen esta localizado a nivel del cromosoma 21q22. Se ha observado que el RFC esta aumentado en tejidos como la mucosa del tracto gastrointestinal, lo que favorece la toxicidad asociada a MTX a este nivel, manifestándose como mucositis. Considerando esto, es destacable la baja incidencia de mucositis severa (16,1\%) que presentaron nuestros pacientes que recibieron MTX comparado con resultados reportados ${ }^{24}$.

El efecto tóxico del MTX podría ser menor por la administración de dosis relativamente altas de leucovorina o ácido folínico (fármaco utilizado para inhibir la toxicidad hematológica y de mucosas relacionada con el MTX) en los pacientes incluídos en el Protocolo 92. En este protocolo, la terapia de rescate con leucovorina se realizaba en base a los niveles de creatinina plasmática, sin embargo, se realizó un estudio de niveles séricos de metotrexato en un grupo de pacientes con LLA sin SD, que reveló que en $50 \%$ de los casos las dosis de leucovorina administradas eran mayores a las recomendadas según estos niveles ${ }^{25}$. Por otro lado, 33/36 niños con LLA y SD evaluados en esta serie recibieron dosis de $1-2 \mathrm{~g} / \mathrm{m}^{2}$, consideradas de nivel intermedio lo que se ha asociado a menor toxicidad ${ }^{26}$; en este grupo de pacientes no fue necesario modificar las dosis de MTX.

Es conocida la mayor susceptibilidad a infecciones en pacientes con SD, debido a disminución de los niveles de enzimas encargadas de producción de radicales libres de oxígeno en los neutrófilos produciendo disminución de su capacidad bactericida. Estos pacientes también presentan disminución de inmunoglobulinas circulantes y disfunción de linfocitos B y $\mathrm{T}$, todo lo que condiciona un estado de inmunodeficiencia relativa que se vería agravado con el uso de citostáticos. Estas alteraciones podrían explicar en nuestro grupo la alta mortalidad relacionada a infección en las fases del protocolo en que no se administró MTX e incluso en pacientes que se encuentran fuera de tratamiento y también la diferencia con pacientes sin SD que utilizan los mismos proto$\operatorname{colos}^{27-29}$.

Aunque se observa una SLE menor en los pacientes con SD que presentan LLA, este grupo debe recibir el mismo tratamiento para LLA que los pacientes sin SD, ya que tienen una respuesta adecuada a la quimioterapia. Por otro lado, la baja incidencia de mucositis se- 
vera y la baja toxicidad asociada a MTX en las dosis usadas, demuestran que es posible utilizar este fármaco sin reducir más su dosis asegurando un tratamiento óptimo para LLA.

En este estudio confirmamos que los niños con SD y LLA tienen características biológicas diferentes a los niños con LLA sin SD, en especial ausencia de casos en menores de un año, ausencia de LLA de estirpe $\mathrm{T}$ y ausencia de translocaciones $(9 ; 22)$ y t $(4 ; 11)$ cuya relación con la patogénesis de la LLA es aún desconocida $^{17-20}$. Si consideramos que las infecciones son la principal causa de mortalidad en los pacientes con SD en tratamiento por LLA, debemos implementar estrategias que logren disminuir estos eventos como: detección precoz de infecciones, utilización de terapias antibióticas más agresivas, evaluación del uso de gammaglobulina de acuerdo al nivel de inmunoglobulinas al inicio y durante el tratamiento y optimizar la terapia de sostén en Unidades de oncología y de pacientes críticos.

\section{Instituciones participantes}

Hospital Luis Calvo Mackenna, Drs: H. del Pozo, V. Beresi, P. Dalborgo, J. Quintana, M. Villarroel, E. Concha, K. Kopp, C. Paris, J. Palma, P. Catalán.

Hospital Roberto del Río, Drs: E. Emparanza, J. Vildósola, P. Joannon, J. Tordecilla, M. Campbell, C. Rizzardini, N. Rodríguez, X. Espinoza.

Hospital Exequiel González Cortés, Drs: R. Fuentes, F. Weinstein, C. Salgado, P. Álvarez, V. Oyarce, A. Figueroa.

Hospital Regional Concepción, Drs: D. Ramos, J. Rojas, E. Fernández, P. Lefourt.

Hospital Gustavo Fricke Viña del Mar, Drs:

S. Tapia, L. Neira, P. Díaz.

Hospital Regional Valdivia, Drs: P. Zolezzi, G. Cea, I. Folatre, M. Yilorm, P. Martínez.

Hospital Sótero del Río, Drs: A. Becker, R. Moreno, M. Fernández, A. Lastra.

Hospital San Juan de Dios, Drs: L. Vargas, H. García, M. Varas, V. Pérez, P. Kabalán, P. Quijarro, D. Álvarez.

Hospital Regional Temuco, Drs: F. Pesse, E. Paez, M. Arriagada.
Hospital San Borja, Drs: P. Advis, I. Ocheretin, P. Silva, F. Espinoza.

Hospital Higueras Talcahuano, Drs: M. Obando, C. Gajardo. buena.

Hospital Talca, Drs: N. Moline, C. Noram-

Hospital Regional de Antofagasta, Dr. A. Urrutia.

\section{Referencias}

1.- Avet Loiseau H, Mechinaud F, Harousseau JL: Clonal hematologic disorders in Down syndrome. J Pediatr Hematol Oncol 1995; 17: 19-24.

2.- Robison LL, Nesbit ME Jr, Sather HN, et al: Down syndrome and acute leukemia in children: a 10-year retrospective survey from Childrens Cancer Study Group. J Pediatr 1984; 105: 235-42.

3.- Margolin J, Steuber C, Poplack D: Acute Lymphoblastic leukemia. En Pizzo P. Poplack D. Principles and practice of pediatric oncology $4^{\text {th }}$ Edition Philadelphia. Lippincott Williams and Wilkins, 2002; 489-544.

4.- Zwann MC, Reinhardt D, Hitzler J: Acute Leukemias in Children with Down Syndrome. Pediatr Clin North Am 2008; 55: 53-70.

5.- Kalwinsky DK, Raimondi SC, Bunin NJ, et al: Clinical and biological characteristics of acute lymphocytic leukemia in children with Down syndrome. Am J Med Genet Suppl 1990; 7: 267-71.

6.- Zipursky A, Poon A, Doyle J: Leukemia in Down syndrome. Pediatr Hematol Oncol 1992; 9: 139-49.

7.- Schrappe M, Reiter A, Zimmermann M, et al: Longterm results of four consecutive trials in childhood ALL performed by the ALL-BFM study group from 1981 to 1995. Leukemia 2000 Sep 14, 2205-22

8.- Silverman LB, Declerck L, Gelber RD, et al: Results of Dana-Farber Cancer Institute Consortium protocols for children with newly diagnosed acute lymphoblastic leukemia (1981-1995). Leukemia 2000 Sep 14, 2247-56.

9.- Pui C-H, Boyett JM, Rivera GK, et al: Long-term results of Total Therapy studies XI, XII and XIIIA for childhood acute lymphoblastic leukemia at St Jude Children's Research Hospital. Leukemia 2000 Sep 14, 2286-94.

10.- Conter V, Arico M, Valsecchi MG, et al: for the Associazione Italiana di Ematologia ed Oncologia Pediatrica (AIEOP). Long-term results of the Italian Association of Pediatric Hematology and Oncology (AIEOP) Acute Lymphoblastic Leukemia Studies, 1982-1995. Leukemia 2000 Sep 14, 2196-204.

11.- Campbell M, Salgado C, Quintana J, et al: Improved 
Outcome for Acute Lymphoblastic Leukemia in Children of a Developing Country: Results of the Chilean National Trial PINDA 87. Medical and Pediatric Oncology 1999; 33: 88-94 (Publicación simultánea Rev Chil Ped 1999; 70: 405-14).

12.- Campbell M: Risk adapted diagnosis and treatment of ALL in South America. $41^{\text {st }}$ Annual Meeting of the International Society of Pediatric Oncology. Symposia Lecture. Blood and Cancer 2009; 53:709 (abstract).

13.- Ragab AH, Abdel Mageed A, Shuster JJ, et al: Clinical characteristics and treatment outcome of children with acute lymphocytic leukemia and Down's syndrome. Cancer 1991; 67: 1057-63.

14.- Whitlock JA, Sather HN, Gaynon P, et al: Clinical characteristics and outcome of children with Down syndrome and acute lymphoblastic leukemia: a Children's Cancer Group study. Blood 2005; 106 (13): 4043-9.

15.- Bene MC, Castoldi G, Knapp W, et al: Proposal for the immunological classification of acute leukemias. Leukemia 1995; 9: 1783-90.

16.- National Cancer Institute Common Toxicity Criteria: Version 2.0. http:// ctep.cancer.gov/reporting/ctc.htlm

17.- Levitt GA, Stiller CA, Chessells JM: Prognosis of Down's syndrome with acute leukaemia. Arch Dis Child 1990; 65: 212-6.

18.- Whitlock JA, Sather HN, Gaynon P, et al: Clinical characteristics and outcome of children with Down syndrome and acute lymphoblastic leukemia: a Children's Cancer Group study. Blood 2005; 106 (13): 4043-9.

19.- Shen JJ, Williams BJ, Zipursky A, et al: Cytogenetic and molecular studies of Down syndrome individuals with leukemia. Am J Hum Genet 1995; 56: 915-25.

20.- Pui CH, Christ WM, Look AT: Biology and clinical significance of cytogenetic abnormalities in childhood acute lymphoblastic leukemia. Blood 1990; 76: 144963.

21.- Malinge S, Izraeli Sh, Crispino J: Insights into the manifestations, outcomes, and mechanisms of leuke- mogenesis in Down syndrome. Blood 2009; 113 (12): 2619-26.

22.- Dördelmann M, Schrappe M, Reiter A, et al: Down's syndrome in childhood acute lymphoblastic leukemia: clinical characteristics and treatment outcome in four consecutive BFM trials. Berlin-Frankfurt-Münster Group. Leukemia 1998; 12 (5): 645-51.

23.- Arico M, Ziino O, Valsecchi MG, et al: Italian Association of Pediatric Hematology and Oncology (AIEOP). Acute lymphoblastic leukemia and Down syndrome: presenting features and treatment outcome in the experience of the Italian Association of Pediatric Hematology and Oncology (AIEOP). Cancer 2008; 113 (3): 515-21.

24.- Taub J, Yubin G: Down Syndrome, Drug Metabolism, and Chromosome 21. Pediatr Blood Cancer 2005; 44: 33-9.

25.- Joannon P, Oviedo I, Campbell M, Tordecilla J: HighDose Methotrexate Therapy of Childhood Acute Lymphoblastic Leukemia: Lack of Relation Between Serum Methotrexate Concentration and Creatinine Clearance. Pediatr Blood Cancer 2004; 43: 17-22.

26.- Buitenkamp T, Mathot R, Haas V, Pieters R, Zwaan $M$ : Methotrexate-induced side effects are not due to differences in pharmacokinetics in children with Down syndrome and acute lymphoblastic leukemia. Haematologica 2010; 95: 1106-13.

27.- Cossarizza A, Monti D, Montagnani G, et al: Precocious aging of the immune system in Down syndrome: alteration of B lymphocytes, T-lymphocyte subsets, and cells with natural killer markers. Am Med Genet Suppl 1990; 7: 213-8.

28.- Ugazio AG, Maccario R, Notarangelo LD, Burgio GR: Immunology of Down syndrome. Am J Med Genet Suppl 1990; 7: 204-12.

29.- Annerén G, Magnusson CG, Lilja G, Nordvall SL: Abnormal serum IgG subclass pattern in children with Down’s syndrome. Arch Dis Child 1992; 67: 628-31. 